\title{
INDEX TO THE BIBLIOGRAPHY, NAMES
}

Abbott, Andrew, 463

Adler-Cohen, Raya, 306

Agriculture and National Development, 297f.

Agulhon, Maurice, 123

Aguzzi, Luciano, 293

Aijmer, Göran, 473

Albert, Alice J., 147

Alexander, Wendy, 147

Allen, William Sheridan, 483

Altena, L.J., 487

Amid, Mohammad Javad, 303

Anderson, Benedict R. O'G. 302

Anderson, Lisa, 472

Andrew, Donna T., 484f.

The Angry Divide. Social and

Economic History of the Western Cape, 468

Anteghini, Alessandra, 139

Antisemitism. An Annotated Bibliography, 2, 465

Een arbeider is zijn loon waardig. Honderd jaar na Rerum Novarum en Christelijk-Sociaal Congres 1891,487

Arbeiter im 20. Jahrhundert, 481

Archer, John E., 145

Archer, Keith, 469

Arfé, Gaetano, 313

Arkush, David, 301

Arnstein, Walter L., 294

Aschenbrenner, Erwin, 119

Ascher, Abraham, 488

Assmann, Birgit, 294

Attard-Maraninchi, MarieFrançoise, 480

Die Aufklärung in Österreich. Ignaz von Born und seine Zeit, 477

Die Aufstieg der deutschen Arbeiterbewegung, 308

Australian Welfare, $133 \mathrm{f}$.

Aux origines provinciales de la Révolution, 478f.
Averill, C., 132

Aya, Rod, 291

Ayçoberry, Pierre, 309

Ayers, Pat, 125

Bach, Hans, 294

Baczko, Bronislaw, 123

Badia, Gilbert, 306

Bailey, Paul, 473

Bakan, Abigail B., 127f.

Baker, Keith Michael, 123

Balfour, Sebastian, 149

Balsom, Denis, 126

Bands. The Brass Band Movements in the 19th and 20th Centuries, 485

Barber, John, 293

Barkan, Lenore, 132

Barmé, Geremie, 472, 473

Baron, Samuel H., 120

Barrachina, Marie-Aline, 123

Bartosek, Karel, 306

Bartoszewski, WIadysIaw T., 487

Basch, Françoise, 471

Bates, Robert H., 463

Beck, Lois, 472

Beckles, Hilary McD., $296 f$.

Beddard, Robert, 477

Beer, Barrett L., 294

Behagg, Clive, 311

Behrendt, Lutz-Dieter, 294

Beijing Spring, 1989, 300

Beilmann, Christel, 484

Beinin, Joel, 292

Belchem, John, 311

Bell, Lynda S., 132

Bell, P.M.H., 145

Bellaby, Paul, 466

Bellingham, Bruce, 125

Bérard, Ewa, 293

Berding, Helmut, 122

Berelowitch, Alexis, 293

Berezin, Mabel, 137

Berlatsky, Joel, 294

Berreen, Rosemary, 134

Berthet, Dominique, 479

Bertolissi, Sergio, 316
Besant, Annie, 312

Best, Geoffrey, 124

Best, Heinrich, 135

Die Bewegung. Hundert Jahr Sozialdemokratie in Österreich, 137

Bianco, William T., 463

Bibliography of the Communist International (19191979), 465

Billingsley, Phil, $300 \mathrm{f}$.

Biografisch woordenboek van het socialisme en de arbeidersbeweging in Nederland, 3, 4, 148

Blatchford, Robert, 312

Blau, Peter, 465

Blinkhorn, Martin, 126

Block, Fred, 121

Blom, Ida, 125

Blumin, Stuart M., 129

Boberach, Heinz, 479

Bock, Gisela, 291

Boffa, Massimo, 123

Bohac, Rodney, 489

Boivin, Marcel, 139

Bolloten, Burnett, 149

Bond, Terry, 294

Bonwetsch, Bernd, 488

Booms, Brigitte, 476

Booms, Hans, 476

Bordin, Ruth, 129

Boyce, George, 126

Boyer, Alain, 309

Bradbury, Bettina, 293

Braddock, Robert C., 294

Brändli, Sebastian, 151

Brand, Jack, 126

Bravo, Anna, 124

Breiger, Ronald L., 465

Breitenbach, Ester, 147

Breyvogel, Wilfried, 484

Brock, Ditmar, 481

Brody, David, 126

The Broken Mirror. China After Tiananmen, 472

Brook, Timothy, 132

Brower, Daniel R., 151 
Brown, Callum G., 147

Brown, Doug, 291

Brown, Richard, 485

Bruguière, Marie-Bernadette, 293

Brunner, Detlev, 144

Bruschi, C., 307

Bruwier, Marinette, 138

Burchardt, Natasha, 124

Burds, Jeffrey, 489

Burgmann, Verity, 475

Burke, Peter, 122

Bush, Barbara, $126 f$.

Busino, G., 119

Byrnes, Michael T., 472

Cabo, Francisco de, 149

Cahm, Caroline, 292

Calhoun, Craig, 465

Callesen, Gerd, 138

Callinicos, Alex, 463

Campfens, Mies, 148

Canadian and Australian La-

bour History, 292f.

Cannadine, David, 311f.

Carsten, Francis L., $481 f$.

Caton, Steven L., 472

Caulier-Mathy, Nicole, 138

Cavallo, Sandra, 304

Cave, Jane, 149

Centenary Essays on Alfred Marshall, 119

Cerutti, Simona, 304

Chagny, Robert, 478f.

Chan, Anita, 473

Chan, Sucheng, 299

Chandler, James K., 123

Charny, Israel W., 465

Chartres, John, 145

Chea, Boon Kheng, 474f.

Chehabi, H.E., 303

Cherubini, Donatella, 313f.

Chevrier, Yves, 301

China and the West: Ideas and Activists, 472f.

Chinese Local Elites and Patterns of Dominance, 132

The Chinese People's Movement, 301

Chinese Society on the Eve of Tiananmen, 301

Chipman, John S., 119

Chong, Woei Lien, 301

Christiansen, Niels Finn, 137

Church, Roy, 126

Claeys, Gregory, 464

Clemens, Bärbel, 136

Coates, Ken, 485

Cohen, Jerome A., 472
Cohen, Paul A., 301

Cohen, Susan Sarah, 465

Coleman, Peter G., 466

Collin, Bruno, 307

The Colonial Moment in Africa. Essays on the movement of minds and materials, 1900-1940, 295

$46 \mathrm{~g}$ Congreso Internacional de americanistas / 46th International Congress of Americanists Amsterdam Holanda 1988, 127

Connolly, James, 312

Constantine, J. Robert, 298

Contemporary Minority Nationalism, 126

Conway, John S., 483

Conzen, Kathleen Neils, 298

Cook, Karin Schweers, 463

Cordasco, Francesco, 129

Cordillot, Michel, 129

Corner, Paul, 126

Corni, Gustavo, 308

Cosseron, Serge, 309

Cotton, James, 473

Coutouvidis, John, 126

Craig, Gordon, 124

Crips, Liliane, 123

The Culture of the Stalin Period, 314

Curtin, Philip D., 122

Dadrian, Vahakn N., 465

Dabiels, Robert V., 293

Darsa, Jan, 465

Davidson, Alastair, 475

Davies, K.G., 477

Davis, Deborah, 301

De l'exil à la résistance. Réfugiés et immigrés d'Europe Centrale en France 19331945, 306

Deacon, Desley, 134

Dean, Mitchell, 312

Debs, Eugene V., 298

Degl'Innocenti, Maurizio, 148

Desama, Claude, 138

Deutsche und Polen in der Revolution 1848-1849, 476

Dex, Shirley, 466

Dictionary of American Immigration History, 129

Dictionnaire biographique du mouvement ouvrier français, XXXVI, XXXVII, 479

Dictionnaire biographique du mouvement ouvrier inter- national: l'Allemagne, 309

Diehl, Paul F., 464

Dietrich, Donald, 483

Dietrich, Elisabeth, 304

DiMaggion, Paul, 121

Dipper, Christof, 125

Dockès, Pierre, 307

Dodson, Michael, 128

Doherty, John C., 486

Dokumente aus geheimen Archiven, 2, $141 \mathrm{f}$.

Domestic Strategies: work and family in France and Italy $1600-1800,304$

Donham, Donald L., $119 f$.

Draus, Franciszek, 123

Dreijmanis, John, 126

Dresch, Paul, 472

Dreyfus, Michael, 139

Droz, Jacques, 309

Duara, Prasenjit, 132

DuBois, Ellen Carol, 131

Duhm, Rainer, 136

Duin, Pieter van, 468

Duncan, Colin, 291

Duncan, Peter, 126

Dusek, Val, 315

Dziedzic, Eugene E., 131

Echinard, Pierre, 480

Edmonds, W.D., 139

Eisenbach, Artur, 487

Elections, Parties and Political Traditions, 142

Elites and Power in Twentieth-Century Spain. Essays in Honour of Sir Raymond Carr, 149

Elorza, Antonio, $149 \mathrm{f}$

El-Sa'id, Rifa'at, 295

Engbring-Romang, Udo, 120

English rural society, 1500 1800. Essays in Honour of Joan Thirsk, 145

Enloe, Cynthia, 124

Erbaugh, Mary S., 473

Ericksen, Robert P., 483

Esenwein, George Richard, 149,488

Esherick, Joseph W., 132

État-révolutions-idéologies. Actes du Colloque de Rennes (21, 22 Avril 1988), 293

Europa und der "Reichseinsatz", 482

Evans, Grant, 133

Faison, Seth, 301

Fakkar, Rouchdi, 468 
Falter, Jürgen W., 142

Family, Household and Gen- Genevois, Danièle Bussy, 150 der Relations in Latin Ame- Gengembre, Gérard, 123 rica, 468

Farrar, Marjorie Milbank, 479

Fayet-Scribe, Sylvie, 139

Feeley-Harnik, Gilian, 466

Fejtö, François, 293

Felber, Roland, 294

Feldman, Gerald D., $125 f$.

Ferchhoff, Wilfried, 122

Ferleger, Lou, 297f.

Fernandez, Manuel A., 297 150

Ferretti, Maria, 316

Fiedler, Gudrun, 482

Fitzpatrick, Sheila, 293

Flores, Marcello, 293

Fontaine, Laurence, 304

Forbath, William E., 470

Ford, Isabella, 312

Foundations of Arbitration, 134 298, 467

Fracki, Edward, 476

Fragner, Bert G., 474

Frankce, Peter, 140

Elizabeth,

Die Französische Revolution, 476

Frauen und Faschismus in Europa, $122 \mathrm{f}$.

Fremad - ad nye veje, 138

The French Revolution and Gordon, Leonard A., 302

the Creation of Modern Po- Gori, Francesca, 293

litical Culture, 3, 123

Fricke, Dieter, 141f.

Fritsche, Peter, 142

Fröschl, Erich, 137

Frow, Edmund, 312

Frow, Ruth, 312

Fuchs, Rachel G., 125

Fudge, Judy, 293

Führer, Karl Christian, 142

Furet, François, 123

Gabel, Helmut, 137

Gailus, Manfred, 482

Galbraith, John Kenneth, 477

Gall, Lothar, 144f.

Gallissot, René, 306

Gallman, J. Matthew, $129 f$.

Garside, W.R., $145 f$.

Gash, Norman, 294

Gehrmann, Rolf, $309 f$.

Gelderen, Martin van, 291

Gellately, Robert, 309
Goch, Stefan, 142f.

Genocide. A Critical Biblio-

Georgel, Jacques, 293

Gérin, Paul, 138

German Peasants' War,

Essays in Honour of Peter

Gershuny, Jonathan, 466

Geyer, Martin H., 304

Geyer, Michael, 124

Gherardi, Silvia, 136

Gielkens, Jan, 148

Gillis, John R., 124

Gilsenan, Michael, 466

Giorgio, Michela de, 123

Gíslason, Ingolfur V., 147f.

Glénisson, Jean, 123

Gold, Thomas B., 301

Goldie, Mark, 477

Goldman, Merle, 301

Golin, Steve, 130

Gollan, Robin, 292

Gómez-Quiñones, Juan, 298

Goode, Richard, 468

Goodman, David S.G., 472f.

Gordon, Eleanor, 147

Gossman, Lionel, 123

Gould, Jeffrey L., 128

Grange, Henri, 307

Grassi, Ernesto, 120

Grau, Carmen, 149

Gray, Marion W., 143

Gray, Richard, 295

Grevemeyer, Jan-Heeren, 300

Griffiths, Gareth, $485 f$.

Groenveld, Simon, 477

Groys, Boris, 314

Grugel, Jean, 126

Gubin, Eliane, 138

Günther, Hans, 314

Guereña, Jean-Louis, 150

Guerrand, Roger-Henri, 141

Gugerli, David, 151

Guisán, Esperanza, 150

Gunn, Simon, 304

Hachtmann, Rüdiger, 309
Hagelweide, Gert, 135

Hagemann, Karen, 309

Hall, Mitchell K., 130

Hannagan, Michael P., 306

Hanschke, Silke, 136

Hardin, Russell, 463

Hardtwig, Wolfgang, 145

Harmsen, Ger, 148

Harnisch, Hartmut, 125

Harris, James F., 143

Harrison, Joseph, 149

Hart, James Mason, 469

Hartford, Kathleen, 301

Haubelt, Josef, 477

Hauch, Gabriella, 305

Haupt, Heinz-Gerhard, 137

Hautmann, Hans, 304

Havel, Vaclav, 477

Haydu, Jeffrey, 293

Hechter, Michael, 463

Heimer, Carol A., 463

Hein, Dieter, 144

Heine, Jorge, 127

Heinemann, Margot, 146

Hélin, Etienne, 138

Hellfeld, Matthias von, 484

Hellmuth, Eckhart, 137

Hemmersam, Flemming, 138

Henkel, Martin, 483

Henry, Paget, 127

"Heraus mit dem Frauenwahlrecht". Die Kämpfe der Frauen in Deutschland und England um die politische Gleichberechtigung, $135 \mathrm{f}$.

Herbert, Trevor, 485

Herbert, Ulrich, 482

Herold, Marc W., 315

Herrmann, Hans Peter, 463

Herrschaft als soziale Praxis, 466

Herrup, Cynthia, 294

Hertz-Eichenrode, Dieter, 308

Hess, J.C., 487

Hey, David, 145

Heyck, Thomas William, 294

Heywood, Paul, 314

Hicks, George, 472

Hierdeis, Helmwart, 476

Hill, Christopher, 146

Hill, Leonidas E., 483

Hirsch, Herbert, 465

Hölscher, Lucian, 483

Hoffmann-Curtis, Kathrin, 123

Hofmeester, Karin, 476

Holt, Richard, 486 
Holzapfel, Kurt, 294

Homan, A.J.P., 487

Homans, George C., 465

Homberger, Eric, $298 \mathrm{f}$.

Hoston, Germaine A., 301

Houdart, Olivier, 313

Houkes, Jannes, 148

Houmann, Børge, 305

Hourani, Albert, 472

Hsiao, Hsin-Luang Michael, 473

Hudson, Pat, 125

Hughes, Agatha C., 293

Hughes, Thomas P., 293

Humphrey, Sarah, 477

Hunt, Lynn, 140

Husband, William B., 314

Hutchinson, John F., 488

Hyndman, Henry Mayers, 312

Idées économiques sous la

Révolution 1789-1794, 307

Iggers, Georg G., 463

Im Hof, Ulrich, 476

Imhof, Arthur E., $309 \mathrm{f}$.

Immigration Reconsidered, 299

Ingram, Norman, 479f.

International Bibliography of Klare, Michael T., 124

Historical Sciences, Vol. Klönne, Arno, 484

LIV, 1985, 123

Isaac, Rhys, 466

Ismael, Tareq Y., 295

Jahn, Peter, 144

James, Wilmot G., 468

Jamieson, Lynn, 147

Jarausch, Konrad H., 143

Jaun, Rudolf, 151

Jeffery, Tom, 137

Jelin, Elizabeth, 468

Jenkins, Brian, 307

Jennings, Jeremy, 140

Jens, Inge, 484

Jensen, Grethe, 138

Jensen, Niels Ole Højstrup, 138

The Jews in Warsaw, 487

Joefield-Napier, Wallace, 127

Johansson, Ella, 124

Jones, Larry Eugene, 143

Jordi, Jean-Jacques, 480

Joris, Elisabeth, 151

Joynes, James Leigh, 312

Kabeya Muase, Charles, 295

Kahan, Vilem, 465

Kaienburg, Hermann, 310
Kammann, Peter, 470

Kaplan, Marion, 123

Karl Kautsky and the Social

Science of Classical Marxism, 120

Karpinskij, Len, 293

Karsten, Luchien, 123, 148

Karsten, Peter, 124

Katz, Friedrich, 294

Katzer, Nikolaus, 314f.

Kautsky, John H., 120

Kealey, Gregory S., 292f.

Keating, Michael, 126

Kelley, Robin D.G., 470

Kelly, David, 472

Kelly, Patrick, 477

Kennedy, Richard, 133f.

Kerr, David, 308

Kesselman, Amy, 130

Keul, Michael, 123

Khoury, Peter, 134

Khoury, Philip S., 472

Kimmel, Michael S., 120

Kingston-Mann, Esther, 489

Kirby, David, 126

Kirk, Tim, 126

Kitay, Jim, 134

Kittsteiner, Heinz-Dieter, 123

Klönne, Arno, 484
Kloke, Ines E., $309 f$.

Klotz, Gérard, 307

Knaack, Rudolf, 141f.

Knudsen, Jonathan, 143

Kocka, Jürgen, 143

Koistinen, Paul, 124

Kondratieva, Tamara, 151

Konrad, Helmet, 304

Kooiman, Dick, 302

Koponen, Juhani, 296

Koshar, Rudy, 136f.

Kossok, Manfred, 294

Kostiner, Joseph, 472

Kozlov, Nicholas N., 315

Kreissler, Felix, 476

Kriegel, Annie, 307

Krishna, C.S., 132

Kritik der Sozialgeschichtsschreibung, 463

Kross, Editha, 294

Künzle, Daniel, 151

Küppers, Steffen, 474

Küttler, Wolfgang, 294

Kuhn, Axel, 476

Kuiper, D.Th., 487

Kuper, Leo, 465

Kurgan-van Hentenryk, Ginette, 126
Kuromiya, Hiroaki, 126

Kurz, Thomas, 483

La Botz, Dan, 130

Labour in the South Pacific, $475 \mathrm{f}$.

Laer, Hermann von, 125

Lambert, Marc, 300

Lammers, Karl Christina, 137

Landau, Karl-Heinz, 136

Lane, Tony, 312

Langewiesche, Dieter, 143

Langford, Paul, 137

Language and Rhetoric of the Revolution, 140

Lannon, Frances, 149

Lansberger, Stefan R., 301

Lapidus, Ira M., 472

Larkin, Emmet, 306

Laville, Jean-Louis, 136

Lechner, Jan, 127

Leckie, Jacqueline, 475f.

Lee, Jenny, 475

Lee, W.R., 125

Lefort, Claude, 123

Lehnert, Detlef, 304

Lenger, Friedrich, 145

Lenman, Bruce P., 477

Leroux, Pierre, 464

Levallois, Michel, 468

Levi, Margaret, 463

Leviatin, David, $130 \mathrm{f}$.

Lewin, Moshe, 293, 489

Lewis, Helena, $123 \mathrm{f}$.

Lewis, Michael, 133

Lewis, Vaughan, 127

Lexikon des alten Handwerks, 143

Life and Work History Analyses: Qualitative and Quantitative Developments, 466

The Life and Work of Karl Polanyi, 291

Lih, Lars T., 151f.

Lijphart, Arend, 127

The Limits of Rationality, 463

Lis, Catharine, 125

Livezeanu, Irina, 137

Lo, Fulang, 132

Locke, Ralph P., 468

Lomnitz, Larissa, 468

Loomis, Terence, 134f.

Lopez, Renée, 480

Popinski, Maciej, 149

Loubère, Leo A., 140

Lovejoy, Frances, 134

Lucas, Colin, 123

Lucassen, Leo, 148 
Luebke, Frederick C., 127

Lüdtke, Alf, 466

Lyth, Peter J., 143 f.

McCord, Edward A., 132

MacDougall, Ian, 146f.

McEachern, Doug, 136

Machiavelli and Republicanism, 291

Macintyre, Stuart, 134

MacLachlan, Colin M., 469

Macmillan, Michael, 126

Madsen, Richard, 301

Mageean, Deirdre M., 486

Magnien, Brigitte, 150

Magraw, Roger, 126

Mahood, Linda, 147

Maier, Robert, 315

Maitron, Jean, 309, 479

Malkiel, Theresa Serber, 471

Malone, Henry O., 483

Mandler, Peter, 125

Manent, Pierre, 123

Manicas, Peter T., 124

Manion, Melanie, 300

Mann, Tom, 312

Manstein, Peter, 483

Maquin, Etienne, 480

Maree, Johann, 468

Markey, Ray, 134

Marsh, Catherine, 466

Marsiske, Hans-Arthur, 471

Marsland, Stephen E., 474

Martí, Casimir, 150

Maruyama, Makoto, 291

Masoero, Alberto, 316

Mathis, Franz, 126

Matthews, Robin C.O., 119

Matthias, Erich, 144

Maurice, Jacques, 150

Measuring the Correlates of

War, 464

Meertens, P.J., 148

Mellink, Albert F., 148

Mélonio, Françoise, 123

Merl, Stephan, 125

Merridale, Catherine, 489

Merton, Robert K., 465

Mesner, Maria, 137

Metcalfe, Alan, 486

Meyer, Alfred G., 120

Meyer, Marshall W., 465

Meyering, Anne, 125

Michalet, Charles-Albert, 307

Michel, Joël, 126

Mielke, Siegfried, 144

Migeod, Heinz-Georg, 474

Migrants, Emigrants and Im- migrants, 486

Mikkelsen, Flemming, 138

1886 [Mil huit cent quattrevingt six]. La Wallonie née de la grève?, 138

The Militarization of the Western World, 124

Miller, Gary J., 463

Miller, Kerby A., 299

Milton, Sybil, 465

Mintzel, Alf, 142

Mitchell, Richard, 134, 292

Il mito dell'URSS. La cultura occidentale e l'Unione Sovietica, 293

Mitterauer, Michael, 124

Mixter, Timothy, 489

Möller, Horst, 137

Molnár, Miklós, 486

Montagna, Paul, 121

Mooers, Colin, 466

Moore, Clive, $475 \mathrm{f}$.

Moorhouse, H.F., 471

Morawska, Eva, 299

Morgan, David W., 120

Morgan, Kevin, 146

Morin, E., 119

Morris, William, 312

Morsy, Magali, 468

Morton, A.L., 146

Moses, John A., $120 f$.

Moskit, Marcin, 149

Motofumi, Asai, 472

Müller-List, Gabriele, 483f.

Müller-Luckner, Elisabeth, 308

Munro, Doug, 475f.

Murphy, Francis J., 140

Murphy, Simon, 126

Mutschlechner, Georg, 477

The Myths We Live By, 124

Nathan, Andrew J., 301

Neubeginn bei Eisen und Stahl im Ruhrgebiet, 483f.

Nexøs, Martin Andersen, 305

Nicholls, A.J., 143

Nielsen, Marie, 305

Niermann, Charlotte, 137

Nikolai Ivanovich Bukharin, 315

Nissan, Elizabeth, 304

Noiriel, Gérard, 480

Norwood, Stephen H., 299

Nove, Alex, 293

O'Brien, Denis, 119

O'Day, Alan, 294

Ogilvie, Sheilagh C., 125
Ó Gráda, Cormac, 306

Oi, Jean C., 301

Oksenberg, Michel, 300

Oliveri, Filippo Salvatore, 294

Orr, Linda, 307

Orth-Peine, Hannelore, 310

Os, M. van, 487

Osertag, Heiger, 310

O'Shaughnessy, Laura Nuzzi, 128

Ouedraogo, Jean-Bernard, $467 f$.

Overzicht van de Archieven in het Internationaal Informatiecentrum en Archief voor de Vrouwenbeweging, 466

Owen, Robert, 464

Ozouf, Mona, 123

Pablo, Michel, 313

Packard, Randall M., 296

Palladino, Grace, 299

Panaccione, Andrea, 293

Paperny, Vladimir, 314

Pappi, Franz Urban, 142

Parsa, Misagh, 303

Pastor, Robert, 127

Patmore, Greg, 292

Paton, Rob, 136

Paucker, Arnold, 483

Paul, Gerhard, 144

Payne, Anthony, 127

Peasant Economy, Culture, and Politics of European Russia, 1800-1921, 489

Peet, Richard, 291f.

Peignot, Jerôme, 464

Peled, Yoav, 489

Pennetier, Claude, 479

Pennybacker, Susan, 137

A People's History of Australia since 1788,475

Pera, Pia, 316

Pérez, Mauricio, 294

Perez-Lizaur, Marisol, 468

Perrow, Charles, 121

Peschanski, Denis, 140, 306

Pethybridge, Roger, 315

Petkov, Krastyu, 478

Peuple, mouvement ouvrier, culture dans l'Espagne contemporaine / Pueblo, movimiento obrero y cultura en la España contemporánea, 150

Pfister, Ulrich, 151

Pierenkemper, Toni, $124 f$. 
Pitavy, François, 129

Plowman, David, 134

Pocock, J.G.A., 123, 477

Pohl, Rainer, 484

Polanyi, Karl, 291

Polanyi-Levitt, Kari, 291

The Politics of Hope. The Origins of Socialism in Britain 1880-1914, 312

Polonsky, Anthony, 487

Pomerans, Arnold J., 486

Poni, Carlo, 304

Pooley, Colin G., 486

Popkin, Jeremy D., $140 f$.

Portes, Alejandro, 299

Potier, Jean-Pierre, 307, 464

Potts, Lydia, 294

Poulat, Emile, 139

Preston, Paul, 149

Price, Don C. , 301

Prins, Gwyn, 477

El proceso de 1938 contra el POUM. Barcelona no fué Moscú, 149

Proletariat und bürgerliche Revolution (1830-1917), 294

Pryor, Frederic L., 127

Pudal, Bernard, 141

Puissant, Jean, 126

Pula, James S., 131

Quellen zur Geschichte der deutschen Gewerkschaftsbewegung im 20 . Jahrhundert, 144

Quilliot, Roger, 141

Quinlan, Michael, 134

Radhakrishnan, P., 133

Raggio, Osvaldo, 304

Raison, Timothy, 146

Ralle, Michel, 149f.

Ramer, Samuel C. , 489

Ramos, Ester, 150

Raxhon, Philippe, 138

Raynaud, Philippe, 123

Read, Christopher, 315

Reay, Barry, 146

Recent Historians of Great Britain, 294

Reich, Jens, 477

Reichard, Richard W., 484

Reid, Douglas A., 486

Reinalda, Bob, 148

Reinalter, Helmut, 136, 476 , 477

Reissig, Harald, 145

Reith, Reinhold, 143
Reitmeier, Gabriele, 128

Renaut, Alain, 123

Renshaw, Patrick, 126

Renwick, John, 140

Reulecke, Jürgen, 145

Reuter, Klaus, 484

Revolutionäres Potential in Europa am Ende des Ersten Weltkrieges, 304

The Revolutions of 1688 . The Andrew Browning Lectures 1988,477

Rey-Goldzeiguer, Anny, 468

Reynolds, Siẩn, 147

Richir, Marc, 123

Rion, Pierre, 138

Ritter, Gerhard A., 142, 308

Robertson, Barbara W., 147

Robin, Régine, 314

Robling, Franz Hubert, 137

Rodriguez, Jesus, 150

Roger, Philippe, 140

Rogers, Nicholas, 137

Rogers, Vaughan, 126

Rohe, Karl, 142

Roschke-Bugzel, Sabine, 474

Rosen, Stanley, 301

Rosner, Peter, 291

Ross, Dorothy, 464

Ross, Ellen, 125

Rossi-Laudi, Ferruccio, 121

Rother, Bernd, 144

Rowe, William T., 132

Roycroft, Maureen, $309 \mathrm{f}$.

Rubinstein, Nicolai, 291

Ruiperez, Maria, 150

Ruiz, Vicki L., 131

Russica. Studi e ricerche sulla Russia contemporanea, 316 Ryan, Selwyn, 127

Sabaliūnas, Leonas, 152

Saich, Tony, 301, 473

Les Saint-Simoniens et l'Orient, 468

Salter, Stephen, 126

Salvadori, Massimo L., 120

Samuel, Raphael, 124, 146

Santbergen, René van, 138

Sárkány, Mihály, 291

Sarkar, Bibekbrata, 473

Savigear, Peter, 126

Saxton, Alexander, 471

Sayad, Abdelmalek, 480

Schäfer, Michael, 310

Schierup, Carl-Ulrik, 316

Schlüpmann, Heide, 123

Schmidlechner, Karin 304
Schmitt, Karl, 142

Schoenherr, Richard, 465

Schönhoven, Klaus, 144

Schokkaert, Luc, 478

Scholz, Rüdiger, 463

Schoppa, R. Keith, 132

Schraepler, Ernst, 145

Schrevel, Margreet, 148

Schulze, Winfried, 137

Schurer, Kevin, 486

Schutte, G.J., 487

Schwartz, Olivier, $480 f$.

Schwartz, Peter, 120

Schwartz, Rosalie, 469

Schwartzman, Kathleen C., $487 \mathrm{f}$.

Schweiz im Wandel. Studien zur neueren Gesellschaftsgeschichte. Festschrift für Rudolf Braun zum 60. Geburtstag, 151

Scott, Tom, 482

Scott, W. Richard, 465

Scribner, Bob, 482

In Search of a Liberal Germany, 143

Seigel, Jerrold, 123

Senlabre Llabata, Carmen, 150

Servet, J.-M., 307

Sewell, William H., Jr, 123

Shaw, George Bernard, 312

Shell, Robert, 468

Sheridan, Tom, 475

Shklar, Judith, 291

Sider, Gerald, 466

Siegel-Wenschkewitz, Leonore, $122 f$.

Siegrist, Hannes, 151

Simons, Mary, 468

Sinclair, Louis, 152

Singer, J. David, 464

Sipos, Péter, 313

Skinner, Quentin, 291

Smith, Dai, 486

Smith, Roger W., 465

Smyth, James J., 147

Social Democratic Federation, 312

Socialist Group of the London Society of Compositors, 312

Solano, Wilebaldo, 149

Soly, Hugo, 125

Soto, Shirlene, 297

Spencer, Jonathan, 304
Spitzer, Leo, 467
M., Splintered Classes. Politics and the Lower Middle Clas- 
ses in Interwar Europe, The Transformation of Politi- Weber, Hermann, 144 $136 f$.

Sport and the working class in modern Britain, 486

Spring in Winter. The 1989 revolutions, 477

Squires, Mike, 312

Sri Lanka, 304

Stadt und Bürgertum im 19. Jahrhundert, $144 f$.

Stanziani, Alessandro, 316

Stapelfeldt, Gerhard, 297

Steila, Daniela, 292, 316

Stephenson, Jayne D., 147

Stevenson, John, 126

Stewart, C.C., 295

Stinas, Agis, 313

Stinchcombe, Arthur L., 463

Stirrat, R.L., 304

Stolcke, Verena, 468

Stone, Carl, 127

Stovall, Tyler, 307

Stoye, John, 477

Strand, David, 132

Strikwerda, Carl, 137

Structures of capital, 121 cal culture. England and Germany in the Late Eighteenth Century, 137

Tranter, Neil L., 486

Traube, Elizabeth, 466

Traverso, Enzo, 121

Weber, Petra, 294f

Weber-Felder, Ulrike, 305

Weinberger, Barbara, 313

Weisbrod, Bernd, 126

Weitz, Eric D., 315

Tribes and State Formation in Welcker, Johanna M., 148 the Middle East, 472

Tripp, Anne Huber, 131

Troch, Harald, $477 \mathrm{f}$.

Trocki, Carl A., 303

Trotter, Joe William, Jr, 299f.

Tucker, M.J., 294

Wells, H.G., 312

Wenk, Silke, 123

Wenzel, Hartmut, 484

Wheeler, Lorraine, 134

Whitaker, John K., 119

Tuñon de Lara, Manuel, 150

Whitaker, Mark P., 304

Uhlenbruch, Bernd, 314

Whitehead, Laurence, 127

Unequal Sisters. A Multicul- Wickert, Christl, 135f. tural Reader in U.S. Wo- Wilding, Peter, 137f. men's History, 131

Unger, Jonathan, 473

Urban, Jan, 477

Useem, Michael, 121

The Uses of Charity, 125 straint, 465

Stuchlik, Gerda, 122f.

Suhr, Gerald D., 489

Sullivan, Lawrence R., 300

Takahashi, Makoto, 293

Talha, Larbi, 308

Tanner, Jakob, 151

Tapper, Richard, 472

Taubert, Friedrich, 481

Taylor, Lewis, 470

Taylor, Michael, 463

Teich, Mikulaš, 477

Temime, Emile, 480

Tenfelde, Klaus, 125f., 144, 304,481

Tennekoon, Serena, 304

Thelen, A.A.J., 487

Thing, Morten, 138, 305

Thirkell, John E.M., 478

Thirsk, Joan, 145

Thompson, Doug, 486f.

Thompson, Paul, 124

Thompson, Willie, 146

Thorndike, Tony, 127

Thurner, Erika, 305

Tibi, Bassam, 472

Valelly, Richard M., 472

Van Damme, Dirk, 137

Vávra, Jaroslav, 477

Venturi, Antonello, 316

Verdoodt, F.J., 487

Verret, Michael, 467

Vidal-Naquet, P., 199

Vincent, Mary, 149

Viroli, Maurizio, 291

Vissol, Thierry, 307

Vogel, Ezra F., 301

Voices from the Hunger Marches, I, 146f.

Von Hagen, Mark, 316

Vovelle, Michel, 478f.

Wilk, Mariusz, 149

Will, Pierre-Etienne, 132

Williams, Howard, 126

Willoughby, John, 315

Wilson, Kathleen, 137

Windhorst, Hans Wilhelm, 125

Winkler, Heinrich August, 483

Wintrich, Herbert, $309 f$.

Wismann, Heinz, 123

Wissell, Rudolf, 145

Witt, Peter-Christian, 142, 308

Woggon, Helga, $138 \mathrm{f}$.

Wojciechowski, Marian, 476

Women and Gender in South. ern Africa to 1945,296

Women's work and the family economy in historical perspective, 125

Woodiwiss, Anthony, 131

Woolf, Stuart, 304

Worden, Andrea, 473

Wagner, Peter, 121f.

Waldinger, Roger, 121

Walker, Cherryl, 296

Walker, Jan, 134

Walle, Heinrich, 483

Wallerstein, Immanuel, 463

Walsted, Anne-Lise, 138

Walter, Eric, 140

Walton, John K., 147

Wasserstrom, Jeffrey N., 301

Waters, Chris, 147

Watson, Michael, 126

Tillman, Hoyt Cleveland, 301 Watson, Rubie S., 132

Tilly, Charles, 299

Tilton, Tim, $150 \mathrm{f}$.

Wcislo, Francis William, 152

Wearing, Michael, 134

Webb, Sidney James, 312

Topham, Tony, 485

Weber, Florence, 141

Workers, Owners and Politics in Coal Mining, 125f.

The Working Class and Politics in Europe and America, $1929-1945,126$

The World is Ill Divided. Women's work in Scotland in the nineteenth and early twentieth centuries, 147

Worobec, Christine D., 489

Wrigley, C.C., 295

Wrigley, Chris J., 147, 313

Wuthnow, Robert, 467

Wynot, Edward D., Jr, 487

Yans-McLaughlin, Virginia, 299 
Young, Lawrence A., 465

Zarrow, Peter, $301 \mathrm{f}$. Zaslavsky, Victor, 293

Zdatny, Steven, 137, 308

Zelin, Madeleine, 132
Zeller, Alfred, 478

Zeuske, Max, 294

Zienkowska, Krystyna, 487

Ziolkowski, Janusz, 477

Zoitl, Helge, 137
Zolberg, Aristide R., 299 Zollitsch, Wolfgang, 311

Zukin, Sharon, 121

Zunz, Olivier, 300

Zwahr, Helmut, 294 